Article

\title{
Clinical Utility of LCT Genotyping in Children with Suspected Functional Gastrointestinal Disorder
}

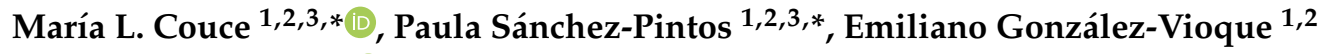 \\ and Rosaura Leis $1,3,4$ (D) \\ 1 Department of Pediatrics, University Clinical Hospital of Santiago de Compostela, IDIS-Health Research \\ Institute of Santiago de Compostela, 15704 Santiago de Compostela, Spain; \\ emiliano.gonzalez.vioque@sergas.es (E.G.-V.); mariarosaura.leis@usc.es (R.L.) \\ 2 CIBERER, Instituto Salud Carlos III, 28029 Madrid, Spain \\ 3 Department of Pediatrics, Universidad de Santiago de Compostela, 15704 Santiago de Compostela, Spain \\ 4 CIBEROBN, Instituto Salud Carlos III, 28029 Madrid, Spain \\ * Correspondence: maria.luz.couce.pico@sergas.es (M.L.C.); paula.sanchez.pintos@sergas.es (P.S.-P.); \\ Tel.: +34-981950151 (M.L.C.); +34-981950134 (P.S.-P.)
}

Received: 21 July 2020; Accepted: 28 September 2020; Published: 1 October 2020

\begin{abstract}
Genetic testing is a good predictor of lactase persistence (LP) in specific populations but its clinical utility in children is less clear. We assessed the role of lactose malabsorption in functional gastrointestinal disorders (FGID) in children and the correlation between the lactase non-persistence (LNP) genotype and phenotype, based on exhaled hydrogen and gastrointestinal symptoms, during a hydrogen breath test (HBT). We also evaluate dairy consumption in this sample. We conducted a 10-year cross-sectional study in a cohort of 493 children with suspected FGID defined by Roma IV criteria. Distribution of the C/T-13910 genotype was as follows: CC, $46.0 \%$; TT, $14.4 \%$ (LP allele frequency, $34.1 \%$ ). The phenotype frequencies of lactose malabsorption and intolerance were $36.3 \%$ and $41.5 \%$, respectively. We observed a strong correlation between genotype and both lactose malabsorption (Cramér's V, 0.28) and intolerance (Cramér's V, 0.54). The frequency of the LNP genotype $(p=0.002)$ and of malabsorption and intolerance increased with age $(p=0.001$ and 0.002 , respectively). In $61 \%$ of children, evaluated dairy consumption was less than recommended. No association was observed between dairy intake and diagnosis. In conclusion, we found a significant correlation between genotype and phenotype, greater in older children, suggesting that the clinical value of genetic testing increases with age.
\end{abstract}

Keywords: lactose intolerance; lactose malabsorption; C/T-13910 polymorphism; functional gastrointestinal disorders

\section{Introduction}

The digestion of lactose, a common disaccharide in human nutrition, is dependent on intestinal lactase (LCT) activity. This enzyme, also known as lactase-phlorizin hydrolase, is a $\beta$-d-galactosidase found in the apical surface of the intestinal microvilli. LCT expression begins before birth, remains high during the nursing period, and then, in most people, progressively declines after weaning, resulting in lactase nonpersistence (LNP) [1]. This genetically programmed reduction in lactase activity varies greatly between populations, with lowest levels observed in Nordic populations ( $<5 \%$ in Denmark) and highest in Korean and Han Chinese populations (approaching 100\%). Large variations are also observed at the regional level [2], affecting about $70 \%$ of the adult population globally. Positive selection of lactase persistence (LP) is usually explained by the gene-culture coevolution hypothesis, whereby LP confers a nutritional advantage in pastoral populations [3,4], and by the calcium assimilation 
hypothesis, which proposes a selective benefit of LP in environments with low ultraviolet irradiation and low dietary vitamin D intake (e.g., high-latitude regions) [5]. LP is common in people of European ancestry, especially in northwestern Europe, and declines in frequency further south and east.

In Caucasians, differences in lactase activity have been linked to two single-nucleotide polymorphisms (SNPs), C/T-13910 and G/A-22018, both of which are located upstream of the LCT-encoding gene [6]. Both variants are in strong linkage disequilibrium, and functional evidences indicates that $\mathrm{C} / \mathrm{T}-13910$ variant is responsible for LP. In Caucasians, the CC and GG variants are associated with hypolactasia and are good predictors of the decrease in intestinal lactase expression, whereas TT and AA- genotypes are predictors of LP. Genetic LP is considered a dominant genotype and CT and GA carriers present intermediate levels of lactase expression. LNP results from the accumulation of transcriptionally suppressive epigenetic changes in haplotypes carrying the SNP C $(-13,010)$ allele, whereas in $T(-13,910)$-containing haplotypes epigenetic inactivation is avoided, facilitating LP [7]. The distribution of these different lactase phenotypes in human populations is highly variable [8]. Spanish series have reported frequencies of the LCT persistence allele ranging from $36.8 \%$ to $66 \%$ [9-12].

LP is one of the most influential factors affecting human dietary patterns [13], and it has been associated with several potential risks in adults, including a higher body mass index (BMI) in European LP populations $[11,14,15]$, especially those that consume large amounts of dairy products $[11,16]$, and metabolic syndrome [10]. Although findings are conflicting, LP is also proposed to influence bone health and fracture risk [17-19].

Several reports [20-24] have evaluated the clinical value of genetic testing, which can be a good predictor of LP/LNP in specific populations. A high correlation between LP and the standard hydrogen breath test (HBT) has also been demonstrated [21,24,25], even though the utility of this test may be limited by ethnicity [23]. Concordance between these two diagnostic tools appears to be influenced by age [26], with greater correlation observed in those over 30 years [20]. The pediatric clinical value of genetic testing and its correlation with HBT findings in childhood requires further evaluation, since most comparative genotyping studies have been performed in adult populations.

In this study, we assessed the role of lactose malabsorption in functional gastrointestinal disorders (FGID) in children, and examined the correlation between the LNP genotype and phenotype, defined based on exhaled hydrogen (H2) measurement and gastrointestinal (GI) symptoms during HBT, in a pediatric population.

\section{Materials and Methods}

\subsection{Study Design and Population}

A cross-sectional, observational, single-center study was performed at the Pediatric Gastroenterology, Hepatology and Nutrition Unit of the University Clinical Hospital of Santiago de Compostela, Spain. The study was approved by the Local Ethics Committee of Santiago-Lugo (code: 2020/319). All procedures were conducted in accordance with the Declaration of Helsinki and signed informed consent was obtained from parents and from children aged $>12$ years.

Between 1 January 2010, and 31 December 2019, 493 consecutive pediatric patients (sample A) with GI symptoms compatible with FGID, defined according Rome IV criteria [27], were evaluated. Exclusion criteria included age $>18$ years and the presence of major metabolic diseases. The study population was subdivided into three age groups: $\leq 5$ years; $6-11$ years; and $\geq 12$ years. The following variables were recorded at recruitment: age; gender; anthropometric data; family history of lactose intolerance; symptoms for which patient consulted; exhaled H2 and GI symptoms during HBT; and genetic study of SNP C/T-13910.

Malabsorption was defined according to HBT test results as a $\mathrm{H} 2$ increase $\geq 20 \mathrm{ppm}$ with respect to baseline, and intolerance was defined as the presence of symptoms during the HBT test. 
In a subgroup of 120 children (sample B), we also analyzed G/A-22018 polymorphisms and serum levels of calcium $(\mathrm{Ca})$, phosphorous $(\mathrm{P})$, parathormone $(\mathrm{PTH})$, calcitonin, calcitriol, and 25-OH vitamin $\mathrm{D}$ (25-OHD), and used a validated questionnaire to collect data on dairy consumption, including the number of weekly milk, cheese, and yogurt servings and the weekly quantity of milk consumed.

All biochemical measurements were obtained from fasting morning plasma samples at the same time of day $(8.00 \mathrm{~h})$ and all patients were free of acute infection and not receiving medication. Reference intervals for biochemical parameters are as follows: Ca: $8.6-10.3 \mathrm{mg} / \mathrm{dL}$ (ages 1-2 years), 9.2-10.3 mg/dL (ages 3-6 years), 8.7-10.5 mg/dL (males aged 6-9 years), 9.0-10.6 mg/dL (females aged 6-9 years), 9.0-10.5 mg/dL (ages 10-14 years), $8.8-10.5 \mathrm{mg} / \mathrm{dL}$ (ages 15-18 years); P: 3.1-5.6 mg/dL; PTH: $12-72 \mathrm{pg} / \mathrm{mL}$; calcitonin: 0-18.2 pg/mL (males), 0-11.5 pg/mL (females); calcitriol: $20-54 \mathrm{pg} / \mathrm{mL}$. 25-OHD levels are considered normal at $>20 \mathrm{ng} / \mathrm{mL}$, while levels of $12-20 \mathrm{ng} / \mathrm{mL}$ and $\leq 12 \mathrm{ng} / \mathrm{mL}$ are considered indicative of 25-OHD insufficiency and deficiency, respectively [28].

\subsection{Anthropometric Measurements}

Standing height was measured with a wall-mounted stadiometer and body weight, to the nearest $100 \mathrm{~g}$, with digital scales. Patients were weighed barefoot after overnight fasting. BMI was calculated as weight $(\mathrm{kg}) /$ height $\mathrm{t}^{2}\left(\mathrm{~m}^{2}\right)$. Subjects were classified according to BMI by using WHO Child Growth Standards for underweight (BMI percentile, $<5$ ), normal weight (BMI percentile, $5-<85$ ), overweight (BMI percentile, 85-94), and obese (BMI percentile, $\geq 95$ ) [29].

Percentiles and z-scores for anthropometric measurements were calculated using the online nutritional assessment tool of the Spanish Society of Gastroenterology, Hepatology, and Nutrition (https://www.seghnp.org/nutricional/) [30].

\subsection{Analytical Measurements}

Concentrations of $\mathrm{Ca}$ and $\mathrm{P}$ were determined by standard procedures with the Advia 2400 Analyzer (Siemens Healthcare Diagnostic Systems, Erlangen, Germany); 25-OHD with the Advia Centaur XP Analyzer (Siemens Healthcare Diagnostics, Erlangen, Germany); PTH with Roche Cobas E 601 (Roche Diagnostic, Indianapolis, IN, USA); calcitonin with INMULITE 2000 (Siemens Healthcare Diagnostics, Erlangen, Germany); and calcitriol by radioimmunoassay in Reference Laboratory S.A. (Hospitalet de Llobregat, Spain).

\subsection{Hydrogen Breath Test}

The HBT test consists of measurement of the concentration of $\mathrm{H} 2$ in exhaled air after $12 \mathrm{~h}$ of fasting and administration of lactose $(2 \mathrm{~g} / \mathrm{kg}$ up to a maximum of $50 \mathrm{~g})$ diluted in aqueous solution $(20 \%)$. Standard requirements for the HBT were applied: absence of treatment in the preceding 15 days with antibiotics, pre/probiotics, or laxatives, or any other drug that could alter the colonic flora; avoidance of fiber-rich food the preceding 3 days; avoidance of physical activity before or during the test; and no smoking prior to testing.

A sample is obtained upon exhaling through the mouthpiece after breathing deeply. The concentration of hydrogen in the exhaled air sample is measured using a gas chromatograph (CM2 MicroLyzer, Quin Tron, Milwaukee, WI, USA). Measurements were taken immediately before ( $0 \mathrm{~min}$, basal measurement) lactose administration and afterwards at 30-min intervals for $3 \mathrm{~h}(30,60,90,120$, 150, and $180 \mathrm{~min}$ ). Peak $\mathrm{H}_{2}$ was recorded.

The results of the HBT were interpreted as follows: absorption (increase $<10 \mathrm{ppm} \mathrm{H}_{2}$ with respect to baseline); poor absorption (increase $\geq 10 \mathrm{ppm}$ and $<20 \mathrm{ppm}_{2}$ with respect to baseline); malabsorption (increase $\geq 20 \mathrm{ppm}_{2}$ with respect to baseline). Exclusion criteria included basal $\mathrm{H}_{2}$ levels $>30 \mathrm{ppm}$. If basal $\mathrm{H}_{2}$ levels were $>10 \mathrm{ppm}$ and $<30 \mathrm{ppm}$, we checked that the measurement at 30 min decrease.

Besides, any GI symptom (abdominal pain, nausea, vomiting, diarrhea) reported by subjects during the test between 0 and 180 min were recorded. 
Tolerance was defined as the absence of symptoms during the test and intolerance as the presence of symptoms during the test.

\subsection{Genetic Study}

Genotyping of C/T-13910 and G/A-22018 SNPs was performed using the SEQUENOM Platform (Agena Bioscience, San Diego, CA, USA).

\subsection{Statistical Analysis}

To verify the homogeneity of distribution of quantitative variables, we used the Student's $t$-test for normally distributed variables, the non-parametric Mann-Whitney $U$ test for variables with a non-normal distribution, and the Kruskal-Wallis test for comparisons between more than 2 groups. Normality of the variables was evaluated using the Shapiro-Wilk statistical test and homoscedasticity using the Bartlett test. In cases in which outliers were detected in the data or the conditions of homoscedasticity were not met, we employed the robust generalization of the Welch test or the Yuen test, using the trimmed mean as an estimator with a cut-off level of 0.2. For analysis of qualitative variables, we used the Chi-square test or Fisher's exact test in cases of non-compliance with the assumptions of the Chi square test.

The degree of association between variables was assessed using Cramér's V test, which returns values between 0 and 1 , where 0 indicates an absence of a relationship and 1 a perfect relationship. Usually accepted cut-off points are as follows: 0.1 , weak relationship; 0.3 , median relationship; and 0.5 , strong relationship.

The agreement between HBT results and those of lactase genotyping was assessed using Cohen's Kappa coefficient. Analyses were performed using R Core Team (version 3.6.3, 2020; R Foundation for Statistical Computing, Vienna, Austria). $p$-values obtained were adjusted using the Bonferroni correction. Only adjusted $p$-values $<0.05$ were considered statistically significant.

\section{Results}

\subsection{Characteristics of the Study Population}

The study population $(n=493)$ (sample A) showed a homogeneous distribution in terms of gender (234 males, 47.4\%). Age distribution was as follows: $\leq 5$ years, $n=50(10.1 \%) ; 6-11$ years, $n=326$ $(66.12 \%) ; \geq 12$ years, $n=117(23.7 \%)$. The characteristics of the study population are summarized in Table 1. The predominant symptom reported on consultation were abdominal pain, diarrhea, and nausea, and the frequency of abdominal pain $(p=0.008)$ and nausea $(p=0.009)$ increased significantly with age.

The C/T-13910 genotype distribution was as follows: CC, $46.0 \%$; CT, 39.5\%; TT $14.4 \%$. The frequency of the LP allele was $34.1 \%$. A significant increase in LNP genotype was observed with increasing age $(p=0.002)$.

\subsection{Phenotype}

The mean frequencies of malabsorption and lactose intolerance in our series were $36.3 \%$ and $41.5 \%$, respectively. With increasing age, the frequency of both parameters increased significantly and progressively (malabsorption, $p=2.2 \times 10^{-16}$; lactose intolerance, $p=0.003$ ). In line with this observation, peak $\mathrm{H}_{2}$ and $\mathrm{H}_{2}$ increase also increased with age $(p<0.001)$ (Table 1$)$.

As reflected in Table 2, despite the higher values obtained for peak and $\mathrm{H}_{2}$ increase in individuals with lactose malabsorption, we found a clear difference for both markers between lactose-intolerant and lactose-tolerant children, with significantly higher values observed in the intolerant group $(p<0.001)$. In lactose-tolerant children aged $>5$ years, we observed a progressive age-associated increase in time to peak $\mathrm{H}_{2}(p=0.005)$. Time to peak $\mathrm{H}_{2}$ was also higher in lactose-intolerant than in lactose-tolerant 
children. The most frequently observed symptom induced by the HBT was abdominal pain, followed by flatulence, diarrhea, and nausea.

Table 1. Characteristics of the study population.

\begin{tabular}{|c|c|c|c|c|c|}
\hline & \multirow{2}{*}{$\begin{array}{l}\text { Subjects } \\
(n=493)\end{array}$} & \multicolumn{3}{|c|}{ Age Groups } & \multirow[t]{2}{*}{$p$} \\
\hline & & $\begin{array}{c}\leq 5 \text { years } \\
(n=50)\end{array}$ & $\begin{array}{c}6-11 \text { years } \\
(n=326)\end{array}$ & $\begin{array}{c}\geq 12 \text { years } \\
(n=117)\end{array}$ & \\
\hline Age $($ mean \pm SD), y & $8.8 \pm 3.3$ & $3.4 \pm 0.9$ & $7.9 \pm 1.9$ & $13.4 \pm 1.31$ & \\
\hline \multicolumn{6}{|c|}{ Anthropometric characteristics } \\
\hline BMI $\left(\mathrm{kg} / \mathrm{m}^{2}\right)$ & $17.86 \pm 3.56$ & $15.51 \pm 1.57$ & $17.16 \pm 3.01$ & $20.62 \pm 3.99$ & $<2.2 \times 10^{-16}$ \\
\hline BMI z-score & $0.28 \pm 1.21$ & $0.12 \pm 1.12$ & $0.32 \pm 1.23$ & $0.26 \pm 1.20$ & 0.446 \\
\hline Underweight & $64[12.9 \%]$ & $8[16 \%]$ & $40[12.2 \%]$ & $17[14.5 \%]$ & \\
\hline Normal weight & $297[60.2 \%]$ & $32[64 \%]$ & $196[60 \%]$ & $69[58.9 \%]$ & 0.983 \\
\hline Overweight & $64[12.7 \%]$ & $5[10 \%]$ & $44[13.4 \%]$ & $15[12.8 \%]$ & \\
\hline Obesity & $68[13.7 \%]$ & $5[10 \%]$ & $47[14.4 \%]$ & $16[13.6 \%]$ & \\
\hline \multicolumn{6}{|l|}{ Family history of LI } \\
\hline Total & 52 [10.5\%] & $1[2 \%]$ & $32[9.8 \%]$ & $19[16.2 \%]$ & \\
\hline Father & $21[4.2 \%]$ & $1[2 \%]$ & $15[4.6 \%]$ & $5[4.2 \%]$ & 0.996 \\
\hline Mother & $19[3.8 \%]$ & 0 & $12[3.6 \%]$ & $7[5.9 \%]$ & \\
\hline Sibling & $12[2.4 \%]$ & 0 & $5[1.5 \%]$ & $7[5.9 \%]$ & \\
\hline \multicolumn{6}{|l|}{ Previous symptoms } \\
\hline Abdominal pain & $277[56.1 \%]$ & $18[36 \%]$ & $194[59.5 \%]$ & $65[55.5 \%]$ & 0.008 \\
\hline Diarrhea & $87[17.6 \%]$ & $11[22 \%]$ & $59[18 \%]$ & $17[14.5 \%]$ & 0.477 \\
\hline Nausea & $34[6.8 \%]$ & 0 & $20[6.1 \%]$ & $14[11.9 \%]$ & 0.009 \\
\hline Vomiting & $57[11.5 \%]$ & $2[4 \%]$ & $43[13.1 \%]$ & $12[10.2 \%]$ & 0.147 \\
\hline Headache & $19[3.8 \%]$ & 0 & $13[3.9 \%]$ & $6[5.1 \%]$ & 0.313 \\
\hline \multicolumn{6}{|l|}{ C/T-13910 genotype } \\
\hline CC & $227[46.04 \%]$ & $12[24 \%]$ & $148[45.3 \%]$ & $67[57.2 \%]$ & 0.002 \\
\hline $\mathrm{CT}$ & $195[39.5 \%]$ & $30[60 \%]$ & $127[38.9 \%]$ & $38[32.4 \%]$ & \\
\hline $\mathrm{TT}$ & $71[14.4 \%]$ & $8[16 \%]$ & $51[15.6 \%]$ & $12[10.2 \%]$ & \\
\hline $\mathrm{C}$ allele frequency & $65.80 \%$ & $54 \%$ & $64.80 \%$ & $73.50 \%$ & \\
\hline $\mathrm{T}$ allele frequency & $34.10 \%$ & $46 \%$ & $35.10 \%$ & $26.40 \%$ & \\
\hline \multicolumn{6}{|l|}{ HBT findings } \\
\hline Lactose absorption & $280[56.7 \%]$ & $42[84 \%]$ & $188[57.6 \%]$ & $50[42.7 \%]$ & $1.47 \times 10^{-5}$ \\
\hline Poor lactose absorption & $34[6.8 \%]$ & $4[8 \%]$ & $21[6.4 \%]$ & $9[7.6 \%]$ & \\
\hline Lactose malabsorption & $179[36.3 \%]$ & $4[8 \%]$ & $117[35.8 \%]$ & $58[49.5 \%]$ & \\
\hline Lactose tolerance & $288[58.4 \%]$ & $36[72 \%]$ & $198[60.7 \%]$ & $54[46.1 \%]$ & 0.003 \\
\hline Lactose intolerance & $205[41.5 \%]$ & $14[28 \%]$ & $128[39.3 \%]$ & $63[53.8 \%]$ & \\
\hline Peak $\mathrm{H}_{2}(\mathrm{ppm})$ & $36.21 \pm 48.1$ & $9.56 \pm 14.98$ & $35.07 \pm 44.1$ & $50.79 \pm 61.20$ & $<0.001$ \\
\hline Time to peak $\mathrm{H}_{2}$ (min) & $88.72 \pm 70.61$ & $71.4 \pm 75.18$ & $87.97 \pm 72$ & $98.20 \pm 63.34$ & $<0.070$ \\
\hline $\mathrm{H}_{2}$ increase (ppm) & $31.87 \pm 47.56$ & $6.18 \pm 14.55$ & $30.66 \pm 43.53$ & $46.23 \pm 60.81$ & $<0.001$ \\
\hline
\end{tabular}

BMI: body mass index; $\mathrm{H}_{2}$ : hydrogen; HBT: hydrogen breath test; LI: lactose intolerance; min: minute; ppm: parts per million; SD: standard deviation; y: years. Data are expressed as the mean \pm SD or as number [\%]. Captions and significant values are in bold.

\subsection{Phenotype/Genotype Correlation}

We observed moderate agreement between HBT and the results of genetic testing (Cohen's Kappa, 0.55; 95\% CI, 0.49-0.61). As shown in Table 3, C/T-13910 polymorphism was significantly correlated with phenotype of absortion/malabsorption by HBT $\left(p<2.22 \times 10^{-16}\right)$ and of lactose tolerance/intolerance $\left(p=2.258 \times 10^{-09}\right)$. Evaluation of the strength of those relationships revealed strong association for malabsorption (Cramér's V, 0.54), and a less strong but non-negligible association for intolerance (Cramér's V, 0.28). It should be noted that $98.8 \%$ of the children with lactose malabsorption were C-allele carriers, and 95.5\% were homozygous for the C-allele. Nonetheless, $215 \mathrm{C}$-allele carriers were identified as lactose absorbers based on HBT results. For both CC and CT genotypes, the frequency of lactose absorbers decreased with increasing age (Table 3). 
Table 2. Comparison of HBT results and induced symptoms in children with lactose malabsorption, tolerance, and intolerance, stratified according to age.

\begin{tabular}{|c|c|c|c|c|c|c|c|c|c|c|c|c|c|c|c|c|}
\hline \multirow{3}{*}{ HBT } & \multicolumn{5}{|c|}{ Malabsorption } & \multicolumn{5}{|c|}{ Tolerance } & \multicolumn{5}{|c|}{ Intolerance } & \multirow[t]{3}{*}{$p^{4}$} \\
\hline & Total & $\leq 5$ years & 6-11 years & $\geq 12$ years & \multirow{2}{*}{$p^{1}$} & Total & $\leq 5$ years & 6-11 years & $\geq 12$ years & \multirow{2}{*}{$p^{2}$} & Total & $\leq 5$ years & 6-11 years & $\geq 12$ years & \multirow{2}{*}{$p^{3}$} & \\
\hline & $(n=179)$ & $(n=4)$ & $(n=117)$ & $(n=58)$ & & $(n=288)$ & $(n=36)$ & $(n=198)$ & $(n=54)$ & & $(n=205)$ & $(n=14)$ & $(n=128)$ & $(n=63)$ & & \\
\hline $\begin{array}{c}\text { Peak } \mathbf{H}_{\mathbf{2}} \\
\text { (ppm) }\end{array}$ & $87.19 \pm 46.9$ & $53.25 \pm 18.5$ & $84.73 \pm 38.4$ & $94.56 \pm 0.9$ & 0.583 & $23.04 \pm 36.5$ & $10.44 \pm 15.6$ & $21.02 \pm 33.7$ & $38.85 \pm 49.5$ & 0.042 & $54.72 \pm 55.8$ & $7.28 \pm 13.4$ & $56.80 \pm 49.3$ & $61.03 \pm 68.3$ & $<0.001$ & $<0.001$ \\
\hline \multicolumn{17}{|l|}{$\begin{array}{c}\text { Time to peak } \\
\mathrm{H}_{2}\end{array}$} \\
\hline$\underset{\text { (Min) }}{(\text { Mean }} \pm S D$ & $134.41 \pm 39.7$ & $165 \pm 17.3$ & $135.38 \pm 40.4$ & $130.34 \pm 38.7$ & 0.736 & $78.22 \pm 72.8$ & $75 \pm 75.04$ & $75.90 \pm 7$ & $88.88 \pm 62.7$ & 0.005 & $103.46 \pm 64.7$ & $62.14 \pm 77.5$ & $106.64 \pm 62.9$ & $106.19 \pm 63.2$ & 0.449 & $1.02 \times 10^{-02}$ \\
\hline $0 \mathrm{~min}$ & 0 & 0 & 0 & 0 & & 96 [33.3\%] & 15 [41.6\%] & $72[36.3 \%]$ & $9[16.6 \%]$ & & 37 [18\%] & $8[57.1 \%]$ & 19 [14.8\%] & 10 [15.8\%] & & \\
\hline $30 \mathrm{~min}$ & $1[0.5 \%]$ & 0 & $1[0.8 \%]$ & 0 & & 40 [13.8\%] & $2[5.5 \%]$ & 29 [14.6\%] & $9[16.6 \%]$ & & $12[5.8 \%]$ & 0 & $9[7 \%]$ & $3[4.7 \%]$ & & \\
\hline $60 \mathrm{~min}$ & $16[8.9 \%]$ & 0 & $11[9.4 \%]$ & $5[8.7 \%]$ & & 15 [5.2\%] & $2[5.5 \%]$ & $8[4 \%]$ & $5[9.2 \%]$ & & $17[3.4 \%]$ & 0 & $11[8.5 \%]$ & $6[9.5 \%]$ & & \\
\hline $90 \mathrm{~min}$ & $27[15 \%]$ & 0 & 16 [13.6\%] & $11[18.9 \%]$ & & $19[6.5 \%]$ & $2[5.5 \%]$ & $12[6 \%]$ & $5[9.2 \%]$ & & $25[12.1 \%]$ & 1 [7.1\%] & 15 [11.7\%] & $9[14.2 \%]$ & & \\
\hline $120 \mathrm{~min}$ & 40 [22.3\%] & 0 & $24[20.5 \%]$ & $16[27.5 \%]$ & & $27[9.3 \%]$ & $4[11.1 \%]$ & $10[5 \%]$ & $13[24 \%]$ & & 29 [14.1\%] & $1[7.1 \%]$ & $20[15.6 \%]$ & $8[12.6 \%]$ & & \\
\hline $150 \mathrm{~min}$ & $43[24 \%]$ & $2[50 \%]$ & $29[24.7 \%]$ & 11 [18.9\%] & & $30[10.4 \%]$ & $4[11.1 \%]$ & $22[11.1 \%]$ & $4[7.4 \%]$ & & $40[19.5 \%]$ & $2[14.2 \%]$ & $25[19.5 \%]$ & $13[20.6 \%]$ & & \\
\hline $180 \mathrm{~min}$ & 53 [29.6\%] & $2[50 \%]$ & $36[30.7 \%]$ & $15[25.9 \%]$ & & $61[21.1 \%]$ & $7[19.4 \%]$ & $45[22.7 \%]$ & $9[16.6 \%]$ & & $45[21.9 \%]$ & $2[14.2 \%]$ & $29[22.6 \%]$ & $14[22.2 \%]$ & & \\
\hline \multicolumn{17}{|l|}{$\mathrm{H}_{2}$ increase } \\
\hline $\begin{array}{c}\text { (Mean } \pm \text { SD } \\
\text { ppm) }\end{array}$ & $82.64 \pm 46.3$ & $50.05 \pm 19$ & $80.29 \pm 37.4$ & $89.60 \pm 60.9$ & 0.623 & $18.85 \pm 35.7$ & $6.88 \pm 15.6$ & $16.91 \pm 32.9$ & $33.92 \pm 48.7$ & 0.025 & $50.17 \pm 55.1$ & $4.35 \pm 11.6$ & $51.92 \pm 49.1$ & $56.79 \pm 68.1$ & $<0.001$ & $<0.001$ \\
\hline$\leq 10 \mathrm{ppm}$ & 0 & 0 & 0 & 0 & & $202[70 \%]$ & $30[83.3 \%]$ & $144[73 \%]$ & 28 [51.8\%] & & $80[39 \%]$ & $13[92.8 \%]$ & 45 [35.1\%] & $22[34.9 \%]$ & & \\
\hline $10-20 \mathrm{pm}$ & $19[10.6 \%]$ & 0 & 0 & 0 & & $23[7.9 \%]$ & $3[8.3 \%]$ & $14[7 \%]$ & $6[11.1 \%]$ & & $13[6.3 \%]$ & 0 & $7[5.4 \%]$ & $5[7.9 \%]$ & & \\
\hline $20-30 \mathrm{pm}$ & $14[7.8 \%]$ & 0 & 14 [11.9\%] & $5[8.7 \%]$ & & $7[2.4 \%]$ & 0 & $6[3 \%]$ & $1[1.8 \%]$ & & $8[3.9 \%]$ & 0 & $5[3.9 \%]$ & $2[3.1 \%]$ & & \\
\hline $30-40 \mathrm{pm}$ & $10[5.5 \%]$ & $1[25 \%]$ & $10[8.5 \%]$ & $5[8.7 \%]$ & & $7[2.4 \%]$ & $1[2.7 \%]$ & $4[2 \%]$ & $2[3.7 \%]$ & & $7[3.4 \%]$ & 0 & $5[3.9 \%]$ & $3[4.7 \%]$ & & \\
\hline $40-50 \mathrm{ppm}$ & $14[7.8 \%]$ & $2[50 \%]$ & $2[1.7 \%]$ & $6[10.3 \%]$ & & $4[1.3 \%]$ & $1[2.7 \%]$ & $1[0.5 \%]$ & $2[3.7 \%]$ & & $6[2.9 \%]$ & $1[7.1 \%]$ & $1[0.7 \%]$ & $4[6.3 \%]$ & & \\
\hline $50-100 \mathrm{ppm}$ & $80[44.6 \%]$ & $1[25 \%]$ & $63[53.8 \%]$ & $29[50 \%]$ & & $32[11.1 \%]$ & $1[2.7 \%]$ & $19[9.5 \%]$ & $12[22.2 \%]$ & & $62[30.2 \%]$ & 0 & $44[34.3 \%]$ & 18 [28.5\%] & & \\
\hline$>100 \mathrm{ppm}$ & $42[23.4 \%]$ & 0 & $30[25.6 \%]$ & 13 [22.1\%] & & $12[4.1 \%]$ & 0 & $10[5 \%]$ & $3[5.5 \%]$ & & $29[14.1 \%]$ & 0 & $20[15.6 \%]$ & $9[14.2 \%]$ & & \\
\hline \multicolumn{17}{|l|}{ Symptoms } \\
\hline $\begin{array}{l}\text { Abdominal } \\
\text { pain }\end{array}$ & 96 [53.6\%] & $1[25 \%]$ & 59 [50.4\%] & $36[62 \%]$ & 0.196 & - & - & - & - & & $165[80.4 \%]$ & 10 [71.4\%] & 101 [78.9\%] & 54 [85.7\%] & 0.340 & \\
\hline Flatulence & 28 [15.6\%] & $1[25 \%]$ & 22 [18.8\%] & $5[8.7 \%]$ & 0.125 & - & - & - & - & & 58 [28.2\%] & $6[42.8 \%]$ & 35 [27.3\%] & 17 [26.9\%] & 0.455 & \\
\hline Diarrhea & 36 [20.1\%] & $4[100 \%]$ & 28 [23.9\%] & $8[13.7 \%]$ & 0.173 & - & - & - & - & & 49 [23.9\%] & $3[21.4 \%]$ & $35[27.3 \%]$ & $11[17.4 \%]$ & 0.308 & \\
\hline Nausea & $16[8.9 \%]$ & $1[25 \%]$ & $10[8.5 \%]$ & $5[8.7 \%]$ & 1 & - & - & - & - & & $4[13.6 \%]$ & $1[7.1 \%]$ & 15 [11.7\%] & $12[19 \%]$ & 0.359 & \\
\hline Vomiting & $3[16.7 \%]$ & $4[100 \%]$ & $2[1.7 \%]$ & $1[1.7 \%]$ & 1 & - & - & - & - & & $4[1.9 \%]$ & 0 & $3[2.3 \%]$ & $1[1.5 \%]$ & 1 & \\
\hline \multicolumn{17}{|l|}{ Symptoms (n) } \\
\hline Mean \pm SD & $1.03 \pm 0.9$ & $0.75 \pm 1.5$ & $1.08 \pm 1.03$ & $0.96 \pm 0.8$ & 0.651 & - & & & & & $1.52 \pm 0.6$ & $1.42 \pm 0.7$ & $1.53 \pm 0.7$ & $1.53 \pm 0.59$ & 0.593 & \\
\hline 1 symptom & $61[34 \%]$ & $1[25 \%]$ & 39 [33.3\%] & $22[37.9 \%]$ & & - & - & - & - & & $117[57 \%]$ & 10 [71.4\%] & 75 [58.5\%] & $32[50.7 \%]$ & $<0.001$ & \\
\hline 2 symptoms & 39 [21.7\%] & 0 & $25[21.3 \%]$ & $14[24.1 \%]$ & & - & - & - & - & & $70[59.8 \%]$ & $2[14.2 \%]$ & $40[31.2 \%]$ & 28 [44.4\%] & $1.02 \times 10^{+03}$ & \\
\hline 3 symptoms & $14[7.8 \%]$ & $3[75 \%]$ & $11[9.4 \%]$ & $2[3.4 \%]$ & & - & - & - & - & & $17[8.2 \%]$ & $2[14.2 \%]$ & $12[9.3 \%]$ & $3[4.7 \%]$ & $<0.001$ & \\
\hline$\geq 3$ symptoms & $1[0.5 \%]$ & 0 & $1[0.8 \%]$ & 0 & & & - & - & - & & $1[0.4 \%]$ & 0 & $1[0.7 \%]$ & 0 & & \\
\hline
\end{tabular}

HBT: hydrogen breath test; min: minutes; $\mathrm{n}$ : number; - : none; ppm: parts per million; SD: standard deviation. Malabsorption is defined as an expired $\mathrm{H}_{2}$ increase $\geq 20$ ppm with respect to baseline, and intolerance as the presence of symptoms during the HBT test. $p^{1}$, comparison between age groups among children with lactose malabsorption; $p^{2}$, comparison between age groups among children with lactose tolerance; $p^{3}$, comparison between age groups among children with lactose intolerance; $p^{4}$, comparison between lactose-tolerant and lactose-intolerant groups. For the malabsorption group, comparisons were made only for children $\leq 12$ years and $>12$ years, as only 4 children $\leq 5$ years presented malabsorption. Captions and significant values are in bold. 
We developed a post-hoc test to examine differences in lactose absorption and lactose tolerance according to C/T-13910 genotype (Figure 1). We observed significant differences in lactose absorption between the CC genotype and the other two genotypes, and in lactose tolerance between each of the three C/T-13190 genotypes analyzed (Supplementary Table S1).

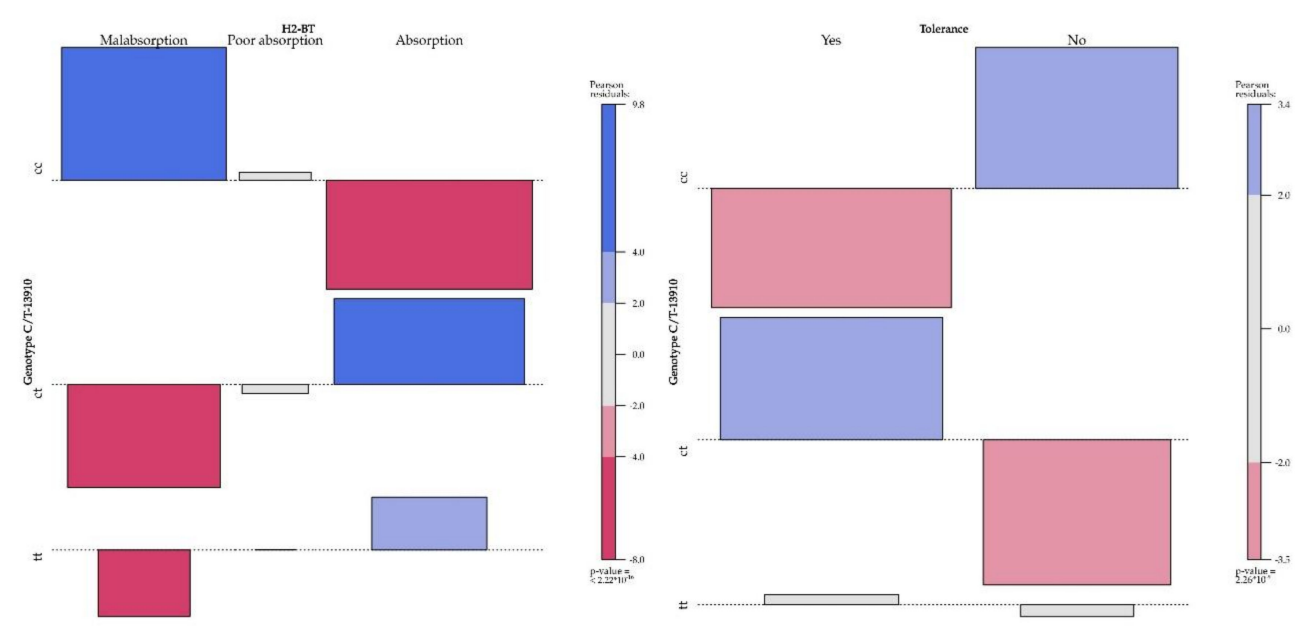

Figure 1. Lactose absorption and tolerance according to C/T-13910 genotype. For the CC genotype, the frequencies of lactose malabsorption and lactose intolerance were higher than expected (positive values shown in blue). Conversely, for the CT genotype, the frequencies of lactose absorption and tolerance were higher than expected.

In sample B, in which we analyzed G/A-22018 polymorphisms, all children with lactose malabsorption were G-allele carriers, and we observed significant differences in lactose malabsorption between the GG genotype and the other two genotypes analyzed. Moreover, there was a strong relationship between C/T-13190 and G/A-22018 polymorphisms ( $p=2.2 \times 10^{-16}$; Cramér's V value, 0.96) (Supplementary Table S1).

\subsection{Dairy Product Intake and Phosphocalcic Metabolism}

As shown in Table 4, evaluation of 120 children using the dairy intake questionnaire revealed that $10.9 \%$ of the study population did not consume milk daily, $15.9 \%$ did not consume yoghurt daily; and $28.4 \%$ did not consume cheese daily. The majority of children, in all age groups, consumed 5-7 servings of milk per week. However, estimated milk consumption varied widely, with a mean weekly consumption of $2604.58 \pm 1477.60 \mathrm{~mL}$. We observed no significant differences in dairy consumption according to age $(p=0.46)$ or polymorphism $(p=0.69)$. The analysis of bone health biomarkers revealed no significant differences between groups with lactose malabsorption, tolerance, and intolerance or between genotypes (data not shown). 
Table 3. C/T-13190 polymorphism and HBT according to age. Genotype-phenotype correlation.

\begin{tabular}{|c|c|c|c|c|c|c|c|c|c|c|c|c|c|c|c|c|}
\hline \multirow{2}{*}{ HBT } & \multicolumn{5}{|c|}{ CC } & \multicolumn{4}{|c|}{$\begin{array}{c}\text { CT-13910 Polymorphism } \\
\text { CT }\end{array}$} & \multicolumn{6}{|c|}{ TT } & \multirow[t]{2}{*}{$p^{4}$} \\
\hline & $\begin{array}{c}\text { Total } \\
(n=227)\end{array}$ & $\begin{array}{l}\leq 5 \text { years } \\
(n=12)\end{array}$ & $\begin{array}{c}6-11 \text { years } \\
(n=148)\end{array}$ & $\begin{array}{c}\geq 12 \text { years } \\
(n=67)\end{array}$ & $p^{1}$ & $\begin{array}{c}\text { Total } \\
(n=195)\end{array}$ & $\begin{array}{l}\leq 5 \text { years } \\
(n=30)\end{array}$ & $\begin{array}{c}6-11 \text { years } \\
(n=127)\end{array}$ & $\begin{array}{c}\geq 12 \text { years } \\
(n=38)\end{array}$ & $p^{2}$ & $\begin{array}{c}\text { Total } \\
(n=71)\end{array}$ & $\begin{array}{c}\leq 5 \text { years } \\
(n=8)\end{array}$ & $\begin{array}{c}6-11 \text { years } \\
(n=51)\end{array}$ & $\begin{array}{c}\geq 12 \text { years } \\
(n=12)\end{array}$ & $p^{3}$ & \\
\hline Absorption & 38 [16.7\%] & 7 [58.3\%] & $24[16.2 \%]$ & $7[10.4 \%]$ & & 177 [90.7\%] & 28 [93.3\%] & 116 [91.3\%] & 33 [86.8\%] & & 65 [91.6\%] & $7[87.5 \%]$ & 48 [94.1\%] & 10 [83.3\%] & & \\
\hline Poor absorption & 18 [7.9\%] & $2[16.6 \%]$ & 12 [8.1\%] & $4[5.9 \%]$ & 0.001 & 11 [5.6\%] & $1[3.3 \%]$ & $7[5.5 \%]$ & $3[7.8 \%]$ & 0.850 & $5[7 \%]$ & $1[12.5 \%]$ & $2[3.9 \%]$ & $2[16.6 \%]$ & 0.412 & $<2.22 \times 10^{-16}$ \\
\hline Malabsorption & $171[75.4 \%]$ & $3[25 \%]$ & 112 [75.6\%] & 56 [83.5\%] & & $7[3.7 \%]$ & $1[3.3 \%]$ & $4[3.1 \%]$ & $2[5.2 \%]$ & & $1[1.4 \%]$ & 0 & $1[1.9 \%]$ & 0 & & \\
\hline Tolerance & $100[44 \%]$ & 11 [91.6\%] & $64[43.4 \%]$ & $25[37.3 \%]$ & & $145[74.3\}$ & $20[66.6 \%]$ & 103 [81.1\%] & $22[57.8 \%]$ & & $43[60.5 \%]$ & $5[62.5 \%]$ & 31 [60.7\%] & 7 [58.3\%] & 1 & \\
\hline Intolerance & $127[56 \%]$ & $1[8.3 \%]$ & 84 [56.7\%] & $42[62.6 \%]$ & 0.002 & $50[25.7 \%]$ & $10[33.3 \%]$ & $24[18.8 \%]$ & $16[42.1 \%]$ & 0.009 & 28 [39.5\%] & $3[37.5 \%]$ & $20[39.2 \%]$ & $5[41.6 \%]$ & & $2.26 \times 10^{-09}$ \\
\hline
\end{tabular}

HBT: hydrogen breath test. Malabsorption is defined as an expired $\mathrm{H}_{2}$ increase $\geq 20 \mathrm{ppm}$ with respect to baseline, and intolerance as the presence of symptoms during the HBT test. $p^{1}$, comparison between age groups among CC subjects. $p^{2}$, comparison between age groups among CT subjects; $p^{3}$, comparison between age groups among TT subjects; $p^{4}$, correlation

between phenotype by HBT and C/T-13910 genotype. Percentages are expressed in brackets. 
Table 4. Dairy product consumption and phosphocalcic metabolism.

\begin{tabular}{|c|c|c|c|c|c|c|c|c|}
\hline & \multirow{2}{*}{ Total $(n=120)$} & \multicolumn{3}{|c|}{ Age Groups } & \multirow{2}{*}{$p^{1}$} & \multirow[t]{2}{*}{$\begin{array}{l}\text { Tolerance } \\
(n=56)\end{array}$} & \multirow[t]{2}{*}{$\begin{array}{c}\text { Intolerance } \\
(n=64)\end{array}$} & \multirow{2}{*}{$p^{2}$} \\
\hline & & $\begin{array}{c}\leq 5 \text { years } \\
(n=6)\end{array}$ & $\begin{array}{c}6-11 \text { years } \\
(n=71)\end{array}$ & $\begin{array}{l}\geq 12 \text { years } \\
(n=43)\end{array}$ & & & & \\
\hline \multicolumn{9}{|l|}{$\begin{array}{c}\text { G/A-22018 } \\
\text { polymorphism }\end{array}$} \\
\hline GG & $84[70 \%]$ & $1[16.6 \%]$ & $47[66.1 \%]$ & $36[83.7 \%]$ & \multirow{3}{*}{$<2.2 \times 10^{-16}$} & $31[55.3 \%]$ & $53[82.8 \%]$ & \multirow{3}{*}{0.002} \\
\hline GA & $29[24.1 \%]$ & $4[66.6 \%]$ & $19[26.7 \%]$ & $6[13.9 \%]$ & & $21[37.5 \%]$ & $8[12.5 \%]$ & \\
\hline $\begin{array}{c}\text { A allele frequency } \\
{[\%]}\end{array}$ & $18 \%$ & $50 \%$ & $20.50 \%$ & $9.40 \%$ & & $25.90 \%$ & $11 \%$ & \\
\hline \multicolumn{9}{|l|}{$\begin{array}{l}\text { Number of dairy } \\
\text { servings/week }\end{array}$} \\
\hline Mean \pm SD & $12.89 \pm 4.89$ & $11.63 \pm 3.14$ & $12.87 \pm 5.13$ & $12.25 \pm 4.71$ & 0.46 & $13.35 \pm 4.92$ & $11.92 \pm 4.80$ & 0.165 \\
\hline $14-17$ & $31[25.8 \%]$ & 0 & $21[29.5 \%]$ & $9[20.9 \%]$ & & $11[19.64 \%]$ & $22[34.38 \%]$ & \\
\hline$>17$ & $14[11.6 \%]$ & 0 & $8[11.2 \%]$ & $6[13.9 \%]$ & & $11[19.64 \%]$ & $1[1.56 \%]$ & \\
\hline \multicolumn{9}{|l|}{$\begin{array}{l}\text { Number of milk } \\
\text { servings/week }\end{array}$} \\
\hline 0 & $13[10.8 \%]$ & 0 & $9[12.6 \%]$ & $4[9.4 \%]$ & 0.643 & $5[8.93 \%]$ & $8[12.5 \%]$ & 0.501 \\
\hline $1-2$ & $6[5 \%]$ & 0 & $5[7.2 \%]$ & $1[2.3 \%]$ & & $3[5.36 \%]$ & $3[4.69 \%]$ & \\
\hline $3-4$ & $7[5.8 \%]$ & 0 & $1[1.4 \%]$ & $6[13.9 \%]$ & & $3[5.36 \%]$ & $4[6.26 \%]$ & \\
\hline $5-7$ & $94[78.4 \%]$ & $6[100 \%]$ & $56[78.8 \%]$ & $32[74.4 \%]$ & & $45[80.36 \%]$ & $49[76.56 \%]$ & \\
\hline \multicolumn{9}{|l|}{$\begin{array}{l}\text { Number of yogurt } \\
\text { servings/week }\end{array}$} \\
\hline 0 & $19[15.8 \%]$ & $1[16.6 \%]$ & $11[15.4 \%]$ & $7[16.2 \%]$ & 0.213 & $8[14.29 \%]$ & $11[17.19 \%]$ & 0.238 \\
\hline $1-2$ & $15[12.6 \%]$ & 0 & $8[11.3 \%]$ & $7[16.2 \%]$ & & 5 [8.93\%] & 10 [15.63\%] & \\
\hline \multicolumn{9}{|l|}{$\begin{array}{l}\text { Estimated weekly } \\
\text { milk intake }\end{array}$} \\
\hline Mean \pm SD & $\begin{array}{c}2604.58 \pm \\
1477.60\end{array}$ & $\begin{array}{c}2154.16 \pm \\
579.56\end{array}$ & $\begin{array}{c}2585.71 \pm \\
1488.12\end{array}$ & $\begin{array}{c}2698.25 \pm \\
1554.54\end{array}$ & 0.972 & $\begin{array}{c}2771.42 \pm \\
1545.62\end{array}$ & $\begin{array}{c}2458.51 \pm \\
1411.40\end{array}$ & 0.459 \\
\hline$\leq 500 \mathrm{~mL}$ & $10[8.3 \%]$ & 0 & $7[9.8 \%]$ & $3[6.9 \%]$ & & $3[5.36 \%]$ & $7[10.93 \%]$ & \\
\hline 500-1000 mL & $10[8.3 \%]$ & 0 & $6[8.4 \%]$ & $4[9.3 \%]$ & & $6[10.72 \%]$ & $4[6.25 \%]$ & \\
\hline $1000-2000 \mathrm{~mL}$ & $23[19.1 \%]$ & $1[16.6 \%]$ & $12[16.9 \%]$ & $10[23.2 \%]$ & & $9[16.08 \%]$ & $14[21.87 \%]$ & \\
\hline $2000-3000 \mathrm{~mL}$ & $36[30 \%]$ & $5[83.3 \%]$ & $22[30.9 \%]$ & $9[20.9 \%]$ & & $16[25.59 \%]$ & $20[31.24 \%]$ & \\
\hline $3000-4000 \mathrm{~mL}$ & $21[17.5 \%]$ & 0 & $12[16.9 \%]$ & $9[20.9 \%]$ & & $9[16.08 \%]$ & $12[18.75 \%]$ & \\
\hline $4000-5000 \mathrm{~mL}$ & $11[9.1 \%]$ & 0 & $8[11.2 \%]$ & $3[6.9 \%]$ & & $7[12.53 \%]$ & $4[6.26 \%]$ & \\
\hline$>5000 \mathrm{~mL}$ & $9[7.5 \%]$ & 0 & $4[5.6 \%]$ & $5[11.6 \%]$ & & $6[10.72 \%]$ & $3[4.68 \%]$ & \\
\hline \multicolumn{9}{|l|}{$\begin{array}{c}\text { Phosphocalcic } \\
\text { metabolism }\end{array}$} \\
\hline Calcium (mg/dL) & $9.79 \pm 0.28$ & $9.73 \pm 0.23$ & $9.82 \pm 0.28$ & $9.74 \pm 0.27$ & 0.401 & $9.81 \pm 0.26$ & $9.77 \pm 0.29$ & 0.308 \\
\hline $\begin{array}{c}\text { Phosphorous } \\
\text { (mg/dL) }\end{array}$ & $4.77 \pm 0.55$ & $4.93 \pm 0.46$ & $4.88 \pm 0.41$ & $4.56 \pm 0.70$ & 0.001 & $4.82 \pm 0.54$ & $4.72 \pm 0.55$ & 0.317 \\
\hline PTH $(\mathrm{pg} / \mathrm{mL})$ & $40.09 \pm 16.64$ & $39.83 \pm 21.02$ & $37.11 \pm 14.03$ & $45.04 \pm 21.02$ & 0.086 & $38.79 \pm 14.38$ & $41.23 \pm 18.44$ & 0.596 \\
\hline $\begin{array}{l}\text { 25-OH vitamin D } \\
(\mathrm{ng} / \mathrm{mL})\end{array}$ & $15.11 \pm 6.24$ & $15.16 \pm 6.30$ & $15.35 \pm 6.70$ & $14.72 \pm 5.51$ & 0.545 & $15.39 \pm 6.21$ & $14.87 \pm 6.30$ & 0.954 \\
\hline Calcitriol $(\mathrm{pg} / \mathrm{mL})$ & $56.15 \pm 15.60$ & $61 \pm 14.54$ & $53.88 \pm 13.03$ & $61 \pm 14.54$ & 0.167 & $60.12 \pm 16.53$ & $52.67 \pm 13.96$ & 0.011 \\
\hline
\end{tabular}

PTH: parathormone. Lactose tolerance is defined as the absence of symptoms during the HBT test and intolerance as the presence of symptoms during it. $p^{1}$, comparison between age groups; $p^{2}$, comparison of lactose tolerant versus lactose intolerant subjects. Percentages are expressed in brackets. Significant values are in bold.

\section{Discussion}

This study of a population of children with FGID symptoms, defined according to Rome IV criteria, reveals a prevalence of lactose malabsorption similar to that of the general population, a high correlation between phenotype and genotype, and low dairy consumption.

Our results show that a diagnosis of lactose malabsorption (defined based on HBT results) was established in about one third (36.3\%) of children with recurrent abdominal pain due to suspected 
functional disorder. This frequency is similar to that reported in other series in children with chronic abdominal pain [31], and in line with that observed in a healthy population in Spain [32]. Moreover, the frequency of malabsorption increased with age, from $8 \%$ in children $\leq 5$ years to $49.5 \%$ in children aged $>12$ years $(p=0.001)$. Lactose malabsorption therefore appears not to play a major role in FGID in younger children, in agreement with previous studies indicating that carbohydrate malabsorption may be an incidental finding in children with FGID, rather than its cause [31]. When evaluating FGID in infants and children, we should screen for celiac disease (CD) as its prevalence among children with irritable bowel syndrome is higher than in general pediatric population [33]. It should also be borne in mind that allergy to cow's milk protein and CD may act as a predisposing or coexisting factor, potentially contributing to inflammation and visceral hypersensitivity in early life, which may manifest as FGID [34,35].

Although several diagnostic tests are available for malabsorption, diagnosis of lactose intolerance remains a challenge [36]. In this study, intolerance was defined based on the presence of symptoms during HBT. Because genetic, enzyme activity, and breath tests only reveal enzyme deficiency, lactose maldigestion, or lactose malabsorption, validated symptom assessment is required to diagnose intolerance [37]. The clinical value of LCT genotype is based on the fact that is a good predictor of LP/LNP in specific populations and shows a good correlation with HBT results. In Caucasians, LP is almost uniformly mediated by the C/T-13910 polymorphism. In this study, we sought to evaluate the clinical value of LCT genotyping in children with GIFD.

Our population had a low prevalence of the LP allele (34.1\%), slightly lower than that reported in other Spanish series [10-12]. Even though we observed a strong correlation between genotype and lactose malabsorption in children $\left(p<2.2 \times 10^{-16}\right.$; Cramér's V, 0.54), in line with the findings of studies in adult populations [20-24], we believe that, from a practical perspective, the most important correlation to evaluate is the extent to which genotype is associated with the presence of GI symptoms. In our series, CC carriers were mainly lactose non-absorbers $(75.4 \%)$, while TT carriers were mainly lactose absorbers (91.6\%). Intermediate lactase expression is the phenotype usually associated with CT heterozygosity. Remarkably, in our population, CT individuals were predominantly lactose absorbers $(90.7 \%)$ and lactose tolerant $(74.3 \%)$, although we observed a slight increase in the frequency of malabsorption and lactose intolerance in CT children with increasing age. This suggests a decline in lactase activity despite the presence of a T allele.

Despite a global significant correlation between C/T-13910 polymorphism and lactose intolerance $\left(p=2.25 \times 10^{-09}\right)$, our results show that the percentage of intolerance among CC children varies from $8.3 \%$ in those $\leq 5$ years to $62.6 \%$ in those $\geq 12$ years, suggesting that genetic testing in younger children could not constitute a practical tool for etiological diagnosis of FIGD. Our findings, in agreement with those of Schirru et al. [38], indicate that the clinical value of genetic testing increases with age.

The SNP G/A-22018 is accepted as being in strong linkage disequilibrium with C/T-13190 [39]. Our results support the recommendation that the detection of a single SNP is sufficient for genetic diagnosis of LP in children. In accordance with our C/T-13910 findings, most children with a GG genotype showed lactose malabsorption (90\%), while almost all AA children were lactose absorbers.

In our population, peak $\mathrm{H}_{2}$ and $\mathrm{H}_{2}$ increase were higher in children with lactose intolerance. This may explain the symptoms observed, as the level of gas production has been linked to the presence and severity of intestinal symptoms [40]. Moreover, in lactose intolerant individuals we observed a significant age-associated increase in $\mathrm{H}_{2}$ production and a parallel increase in the frequency of abdominal pain. One interesting finding is that time to peak $\mathrm{H}_{2}$ was shorter in lactose tolerant than intolerant children. This difference may be linked to an earlier gastric emptying/clearance and shorter intestinal transit times in lactose tolerants, although this hypothesis runs counter to the idea that the rapid movement of hyperosmolar content from the stomach into the intestine causes some of the symptoms identified in lactose intolerant children [41,42], since residual lactase activity is overcome [41,42]. 
We also evaluated dairy consumption, which is one of several interacting factors that give rise to marked interindividual differences in sensitivity to incompletely absorbed carbohydrates, and that influence the development and severity of symptoms in patients with lactose malabsorption [36]. Another aspect that can influence symptoms is the microbiota, as higher levels of Bifidobacterium species have been reported in CC versus TT or CT genotypes in European populations, a difference explained by the greater abundance of lactose available for bacterial fermentation in CC individuals due to lactose malabsorption [43]. Furthermore, probiotic supplementation in lactose-intolerant individuals has beneficial effects on HBT results and on symptoms of lactose intolerance [44].

Contrary to expectations $[45,46]$, we found no significant differences in dairy consumption between lactose tolerant and lactose intolerant children, and no differences in the number of dairy servings per week between genotypes. This finding could be influenced by social, environmental, or behavioral confounding factors, not limited in our study. Even though reduced lactose intake, rather than complete exclusion, is recommended in individuals with lactose intolerance [2], a considerable proportion of our study population did not consume milk or other dairy products daily. This is likely due to several factors, including erroneous attribution of symptoms to lactose intolerance, improper dietary management of lactose intolerance, and the progressive decrease in the consumption of dairy products in the pediatric population in Spain $[47,48]$. Milk constitutes a basic source of dietary calcium in most Western diets, and dairy consumption in $61 \%$ of children evaluated is below the level necessary to achieve the recommended calcium intake [49]. Given that the participants in the present study were evaluated during their growth period, dairy exclusion may have later consequences on phosphocalcic metabolism that were not yet evident in our population, since dairy consumption significantly increases bone mineral content in children [50].

Limitations of our study include methodological limitations inherent to the diagnosis of malabsorption using HBT rather than the gold standard procedure (intestinal biopsy), especially in young children, and the lack of Mendelian randomization in the evaluation of dairy consumption.

\section{Conclusions}

We observed a significant correlation between genotype and phenotype in children with suspected FGID and an increase with age in lactose malabsorption and intolerance (according to HBT results) in LNP subjects. These findings suggest that the practical value of genetic testing is greater in older children.

Supplementary Materials: The following are available online at http://www.mdpi.com/2072-6643/12/10/3017/s1, Supplementary Table S1: Comparison of genotypes according to lactose absorption/malabsorption and lactose tolerance/intolerance.

Author Contributions: M.L.C. and R.L. contributed to the design, methodology and supervision of the study; P.S.-P., M.L.C. and R.L. contributed to data selection and extraction, presentation of the results, and drafting of the manuscript; E.G.-V. contributed to the presentation of the results and drafting of the manuscript. All authors have read and agreed to the published version of the manuscript.

Funding: This research received no external funding.

Conflicts of Interest: The authors declare no conflict of interest.

\section{References}

1. Rasinperä, H.; Kuokkanen, M.; Kolho, K.-L.; Lindahl, H.; Enattah, N.S.; Savilahti, E.; Orpana, A.; Jarvela, I. Transcriptional downregulation of the lactase (LCT) gene during childhood. Gut 2005, 54, 1660-1661. [CrossRef] [PubMed]

2. Misselwitz, B.; Butter, M.; Verbeke, K.; Fox, M. Update on lactose malabsorption and intolerance: Pathogenesis, diagnosis and clinical management. Gut 2019, 68, 2080-2091. [CrossRef] [PubMed]

3. Simoons, F.J. Primary adult lactose intolerance and the milking habit: A problem in biological and cultural interrelations. II. A culture historical hypothesis. Am. J. Dig. Dis. 1970, 15, 695-710. [CrossRef] [PubMed] 
4. McCracken, R.D. Origins and Implications of the Distribution of Adult Lactase Deficiency in Human Populations. J. Trop. Pediatr. Environ. Child. Health 1971, 17, 7-10. [CrossRef]

5. Flatz, G.; Rotthauwe, H. Lactose nutrition and natural selection. Lancet 1973, 302, 76-77. [CrossRef]

6. Enattah, N.S.; Sahi, T.; Savilahti, E.; Terwilliger, J.D.; Peltonen, L.; Jarvela, I. Identification of a variant associated with adult-type hypolactasia. Nat. Genet. 2002, 30, 233-237. [CrossRef]

7. Labrie, V.; Buske, O.J.; Oh, E.; Jeremian, R.; Ptak, C.; Gasiunas, G.; Maleckas, A.; Petereit, R.; Žvirbliene, A.; Adamonis, K.; et al. Lactase nonpersistence is directed by DNA-variation-dependent epigenetic aging. Nat. Struct. Mol. Biol. 2016, 23, 566-573. [CrossRef]

8. Swallow, D.M. Genetics of Lactase Persistence and Lactose Intolerance. Annu. Rev. Genet. 2003, 37, 197-219. [CrossRef]

9. Enattah, N.S.; Trudeau, A.; Pimenoff, V.N.; Maiuri, L.; Auricchio, S.; Greco, L.; Rossi, M.; Lentze, M.; Seo, J.K.; Rahgozar, S.; et al. Evidence of Still-Ongoing Convergence Evolution of the Lactase Persistence T-13910 Alleles in Humans. Am. J. Hum. Genet. 2007, 81, 615-625. [CrossRef]

10. Almon, R.; Álvarez-León, E.E.; Engfeldt, P.; Serra-Majem, L.; Magnuson, A.; Nilsson, T.K. Associations between lactase persistence and the metabolic syndrome in a cross-sectional study in the Canary Islands. Eur. J. Nutr. 2009, 49, 141-146. [CrossRef]

11. Corella, D.; Arregui, M.; Coltell, O.; Portolès, O.; Guillem-Saiz, P.; Carrasco, P.; Sorli, J.V.; Ortega-Azorín, C.; González, J.I.; Ordovás, J.M. Association of the LCT-13910C > T polymorphism with obesity and its modulation by dairy products in a Mediterranean population. Obesity 2010, 19, 1707-1714. [CrossRef]

12. Jiménez, J.L.D.; Suárez, A.F.; Colmenero, A.; Úrsula, M.; Cantillo, D.F.; Pelayo, I.L. Primary hypolactasia diagnosis: Comparison between the gaxilose test, shortened lactose tolerance test, and clinical parameters corresponding to the C/T-13910 polymorphism. Clin. Nutr. 2017, 36, 471-476. [CrossRef] [PubMed]

13. Comerford, K.B.; Pasin, G. Gene-Dairy Food Interactions and Health Outcomes: A Review of Nutrigenetic Studies. Nutrients 2017, 9, 710. [CrossRef] [PubMed]

14. Kettunen, J.; Silander, K.; Saarela, O.; Amin, N.; Müller-Nurasyid, M.; Timpson, N.J.; Surakka, I.; Ripatti, S.; Laitinen, J.; Hartikainen, A.-L.; et al. European lactase persistence genotype shows evidence of association with increase in body mass index. Hum. Mol. Genet. 2010, 19, 1129-1136. [CrossRef] [PubMed]

15. Almon, R.; Álvarez-León, E.E.; Serra-Majem, L. Association of the European Lactase Persistence Variant (LCT-13910 C>T Polymorphism) with Obesity in the Canary Islands. PLoS ONE 2012, 7, e43978. [CrossRef]

16. Lamri, A.; Poli, A.; Emery, N.; Bellili, N.; Velho, G.; Lantieri, O.; Balkau, B.; Marre, M.; Fumeron, F. The lactase persistence genotype is associated with body mass index and dairy consumption in the D.E.S.I.R. study. Metabolism 2013, 62, 1323-1329. [CrossRef]

17. Obermayer-Pietsch, B.M.; Bonelli, C.M.; Walter, D.E.; Kuhn, R.J.; Fahrleitner-Pammer, A.; Berghold, A.; Goessler, W.; Stepan, V.; Dobnig, H.; Leb, G.; et al. Genetic Predisposition for Adult Lactose Intolerance and Relation to Diet, Bone Density, and Bone Fractures. J. Bone Miner. Res. 2004, 19, 42-47. [CrossRef]

18. Enattah, N.; Pekkarinen, T.; Välimäki, M.J.; Löyttyniemi, E.; Jarvela, I. Genetically defined adult-type hypolactasia and self-reported lactose intolerance as risk factors of osteoporosis in Finnish postmenopausal women. Eur. J. Clin. Nutr. 2005, 59, 1105-1111. [CrossRef]

19. Yang, Q.; Lin, S.L.; Yeung, S.L.R.A.; Kwok, M.K.; Xu, L.; Leung, G.M.; Schooling, C.M. Genetically predicted milk consumption and bone health, ischemic heart disease and type 2 diabetes: A Mendelian randomization study. Eur. J. Clin. Nutr. 2017, 71, 1008-1012. [CrossRef]

20. Di Stefano, M.; Terulla, V.; Tana, P.; Mazzocchi, S.; Romero, E.; Corazza, G.R. Genetic test for lactase non-persistence and hydrogen breath test: Is genotype better than phenotype to diagnose lactose malabsorption? Dig. Liver Dis. 2009, 41, 474-479. [CrossRef]

21. Pohl, D.; Savarino, E.V.; Hersberger, M.; Behlis, Z.; Stutz, B.; Goetze, O.; Eckardstein, A.V.; Fried, M.; Tutuian, R. Excellent agreement between genetic and hydrogen breath tests for lactase deficiency and the role of extended symptom assessment. Br. J. Nutr. 2010, 104, 900-907. [CrossRef] [PubMed]

22. Enko, D.; Rezanka, E.; Stolba, R.; Halwachs-Baumann, G. Lactose Malabsorption Testing in Daily Clinical Practice: A Critical Retrospective Analysis and Comparison of the Hydrogen/Methane Breath Test and Genetic Test (C/T-13910 Polymorphism) Results. Gastroenterol. Res. Pract. 2014, 2014, 464382. [CrossRef]

23. Santonocito, C.; Scapaticci, M.; Guarino, D.; Annicchiarico, E.B.; Lisci, R.; Penitente, R.; Gasbarrini, A.; Zuppi, C.; Capoluongo, E. Lactose intolerance genetic testing: Is it useful as routine screening? Results on 1426 south-central Italy patients. Clin. Chim. Acta 2015, 439, 14-17. [CrossRef] [PubMed] 
24. Coluccia, E.; Iardino, P.; Pappalardo, D.; Brigida, A.L.; Formicola, V.; De Felice, B.; Guerra, C.; Pucciarelli, A.; Amato, M.R.; Riegler, G.; et al. Congruency of Genetic Predisposition to Lactase Persistence and Lactose Breath Test. Nutrients 2019, 11, 1383. [CrossRef] [PubMed]

25. Högenauer, C.; Hammer, H.F.; Mellitzer, K.; Renner, W.; Krejs, G.J.; Toplak, H. Evaluation of a new DNA test compared with the lactose hydrogen breath test for the diagnosis of lactase non-persistence. Eur. J. Gastroenterol. Hepatol. 2005, 17, 371-376. [CrossRef]

26. Nagy, D.; Bogácsi-Szabó, E.; Várkonyi, Á.; Csányi, B.; Czibula, Á.; Bede, O.; Tari, B.; Raskó, I. Prevalence of adult-type hypolactasia as diagnosed with genetic and lactose hydrogen breath tests in Hungarians. Eur. J. Clin. Nutr. 2009, 63, 909-912. [CrossRef]

27. Hyams, J.S.; Di Lorenzo, C.; Spas, M.; Shulman, R.J.; Staiano, A.; van Tilburg, M. Functional Disorders: Children and Adolescent. Gastroenterology 2016, 150, 1456-1468.e2. [CrossRef]

28. Munns, C.F.; Shaw, N.; Kiely, M.; Specker, B.L.; Thacher, T.D.; Ozono, K.; Michigami, T.; Tiosano, D.; Mughal, M.Z.; Mäkitie, O.; et al. Global Consensus Recommendations on Prevention and Management of Nutritional Rickets. J. Clin. Endocrinol. Metab. 2016, 101, 394-415. [CrossRef]

29. WHO Multicentre Growth Reference Study Group; Onis, M. WHO Child Growth Standards based on length/height, weight and age. Acta Paediatr. 2007, 95, 76-85. [CrossRef]

30. Nutritional Assessment Tool of the Spanish Society of Gastroenterology, Hepatology, and Nutrition. Available online: https://www.seghnp.org/nutricional/ (accessed on 15 May 2020).

31. Posovszky, C.; Roesler, V.; Becker, S.; Iven, E.; Hudert, C.; Ebinger, F.; Calvano, C.; Warschburger, P. Roles of Lactose and Fructose Malabsorption and Dietary Outcomes in Children Presenting with Chronic Abdominal Pain. Nutrients 2019, 11, 3063. [CrossRef]

32. Leis, R.; Tojo, R.; Pavón, P.; Douwes, A. Prevalence of Lactose Malabsorption in Galicia. J. Pediatr. Gastroenterol. Nutr. 1997, 25, 296-300. [CrossRef] [PubMed]

33. Cristofori, F.; Fontana, C.; Magistà, A.; Capriati, T.; Indrio, F.; Castellaneta, S.; Cavallo, L.; Francavilla, R. Increased prevalence of celiac disease among pediatric patients with irritable bowel syndrome: A 6-year prospective cohort study. JAMA Pediatr. 2014, 168, 555. [CrossRef] [PubMed]

34. Pensabene, L.; Salvatore, S.; D’Auria, E.; Parisi, F.; Concolino, D.; Borrelli, O.; Thapar, N.; Staiano, A.; Vandenplas, Y.; Saps, M. Cow's Milk Protein Allergy in Infancy: A Risk Factor for Functional Gastrointestinal Disorders in Children? Nutrients 2018, 10, 1716. [CrossRef] [PubMed]

35. Valitutti, F.; Fasano, A. Breaking Down Barriers: How Understanding Celiac Disease Pathogenesis Informed the Development of Novel Treatments. Dig. Dis. Sci. 2019, 64, 1748-1758. [CrossRef] [PubMed]

36. Fassio, F.; Facioni, M.S.; Guagnini, F. Lactose Maldigestion, Malabsorption, and Intolerance: A Comprehensive Review with a Focus on Current Management and Future Perspectives. Nutrients 2018, 10, 1599. [CrossRef]

37. Hammer, H.F.; Hammer, J.; Fox, M. Mistakes in the management of carbohydrate intolerance and how to avoid them. UEG Educ. 2019, 19, 9-14.

38. Schirru, E.; Corona, V.; Usai-Satta, P.; Scarpa, M.; Cucca, F.; De Virgiliis, S.; Rossino, R.; Frau, F.; Macis, M.D.; Jores, R.-D.; et al. Decline of Lactase Activity and C/T-13910 Variant in Sardinian Childhood. J. Pediatr. Gastroenterol. Nutr. 2007, 45, 503-506. [CrossRef]

39. Poulter, M.; Hollox, E.J.; Harvey, C.B.; Mulcare, C.; Peuhkuri, K.; Kajander, K.; Sarner, M.; Korpela, R.; Swallow, D.M. The causal element for the lactase persistence/non-persistence polymorphism is located in a 1 $\mathrm{Mb}$ region of linkage disequilibrium in Europeans. Ann. Hum. Genet. 2003, 67, 298-311. [CrossRef]

40. Zhu, Y.; Zheng, X.; Cong, Y.; Chu, H.; Fried, M.; Dai, N.; Fox, M. Bloating and Distention in Irritable Bowel Syndrome: The Role of Gas Production and Visceral Sensation After Lactose Ingestion in a Population With Lactase Deficiency. Am. J. Gastroenterol. 2013, 108, 1516-1525. [CrossRef]

41. Peuhkuri, K.; Vapaatalo, H.; Nevala, R.; Korpela, R. Influence of the pharmacological modification of gastric emptying on lactose digestion and gastrointestinal symptoms. Aliment. Pharmacol. Ther. 1999, 13, 81-86. [CrossRef]

42. Labayen, I.; Forga, L.; Gonzalez, A.; Lenoir-Wijnkoop, I.; Nutr, R.; Martinez, J.A. Relationship between lactose digestion, gastrointestinal transit time and symptoms in lactose malabsorbers after dairy consumption. Aliment. Pharmacol. Ther. 2001, 15, 543-549. [CrossRef] [PubMed]

43. Blekhman, R.; Goodrich, J.K.; Huang, K.; Sun, Q.; Bukowski, R.; Bell, J.T.; Spector, T.D.; Keinan, A.; Ley, R.E.; Gevers, D.; et al. Host genetic variation impacts microbiome composition across human body sites. Genome Biol. 2015, 16, 191. [CrossRef] [PubMed] 
44. Leis, R.; De Castro, M.J.; De Lamas, C.; Picáns, R.; Couce, M.-L. Effects of Prebiotic and Probiotic Supplementation on Lactase Deficiency and Lactose Intolerance: A Systematic Review of Controlled Trials. Nutrients 2020, 12, 1487. [CrossRef] [PubMed]

45. Almon, R.; Sjöström, M.; Nilsson, T.K. Lactase non-persistence as a determinant of milk avoidance and calcium intake in children and adolescents. J. Nutr. Sci. 2013, 2, 1-5. [CrossRef]

46. Alharbi, O.; El-Sohemy, A. Lactose Intolerance (LCT -13910C >T) Genotype Is Associated with Plasma 25-Hydroxyvitamin D Concentrations in Caucasians: A Mendelian Randomization Study. J. Nutr. 2017, 147, 1063-1069. [CrossRef]

47. López-Sobaler, A.M.; Aparicio, A.; Rodríguez, L.G.G.; Cuadrado-Soto, E.; Rubio, J.; Marcos, V.; Sanchidrián, R.; Santos, S.; Pérez-Farinós, N.; Re, M.; et al. Adequacy of Usual Vitamin and Mineral Intake in Spanish Children and Adolescents: ENALIA Study. Nutrients 2017, 9, 131. [CrossRef]

48. Madrigal, C.; Soto-Méndez, M.J.; Hernández-Ruiz, A.; Valero, T.; Ávila, J.M.; Lara-Villoslada, F.; Leis, R.; de Victoria, E.M.; Moreno, J.M.; Ortega, O.M.; et al. Dietary and Lifestyle Patterns in the Spanish Pediatric Population (One to > 10 Years Old): Design, Protocol, and Methodology of the EsNuPI Study. Nutrients 2019, 11, 3050. [CrossRef]

49. EFSA Panel on Dietetic Products, Nutrition and Allergies (NDA). Scientific Opinion on nutrient requirements and dietary intakes of infants and young children in the European Union. EFSA J. 2013, 11, 3408.

50. De Lamas, C.; De Castro, M.J.; Gil-Campos, M.; Gil, Á.; Couce, M.L.; Leis, R. Effects of Dairy Product Consumption on Height and Bone Mineral Content in Children: A Systematic Review of Controlled Trials. Adv. Nutr. 2019, 10, S88-S96. [CrossRef]

(C) 2020 by the authors. Licensee MDPI, Basel, Switzerland. This article is an open access article distributed under the terms and conditions of the Creative Commons Attribution (CC BY) license (http://creativecommons.org/licenses/by/4.0/). 